\title{
Pack ice as a two-dimensional granular plastic: a new constitutive law
}

\author{
Keguang WANG \\ Department of Physical Sciences, University of Helsinki, FIN-00014 Helsinki, Finland \\ E-mail: keguang.wang@helsinki.fi
}

\begin{abstract}
The purpose of this study is to propose a new constitutive law for pack ice, which is not only capable of simulating the in-plane shear and out-of-plane uniaxial compression, but also capable of avoiding overestimating divergence during shear. The pack ice is treated as a two-dimensional granular plastic, obeying Coulomb's friction law with a maximum principal stress limit. During the out-of-plane uniaxial compression process the flow rule is normal, while during the in-plane shear process the flow rule is coaxial with a linearly varying dilatancy angle describing the divergence. The strength parameterizations are based on thickness and compactness of the pack ice; weakening or hardening can be achieved through advection and redistribution.
\end{abstract}

\section{INTRODUCTION}

Pack ice is an aggregate of ice floes drifting on the sea surface. Due to the great difficulty in directly measuring the large-scale ice stress, validation of the mechanical behavior of ice relies on the comparison of the resulting deformation with that observed in the pack ice. A special deformation pattern is the widely observed linear kinematic features (LKFs), which are long, narrow geophysical features with a much higher deformation rate than in the surrounding pack ice. In general, they may consist of open water, new ice, young ice, rafted ice or even ridged ice (Kwok, 2000).

It has been shown that LKF patterns are closely related to the slope of the yield curve by (Erlingsson, 1988; Pritchard, 1988; Wang, 2004)

$$
\frac{\mathrm{d} \sigma_{\|}}{\mathrm{d} \sigma_{\mathrm{I}}}=\cos 2 \theta
$$

where $\sigma_{\mathrm{I}}$ and $\sigma_{\mathrm{II}}$ are the mean compressive stress and maximum shear stress, and $2 \theta$ is the angle between intersecting LKFs with the larger principal stress as the bisector. Typical observed deformation patterns are in-plane shear (e.g. Marko and Thomson, 1977; Erlingsson, 1988; Kwok, 2000), which results in intersecting LKFs in pack ice, and uniaxial compression, which has been widely observed as pressure ridges in the polar and subpolar seas. According to Equation (1), the in-plane shear process is due to the granular flow following Coulomb's friction law, while the out-of-plane uniaxial compression process is due to the limit of maximum principal stress.

Classical sea-ice dynamical models (Coon and others, 1974; Hibler, 1979) do not simulate such features, and most models using Coulomb's law take the limit of maximum compressive stress (e.g. Smith, 1983; Ip and others, 1991; Tremblay and Mysak, 1997; Hibler and Schulson, 2000). However, applying such a limit generally leads to no pressure ridge or two intersecting shear ridges. Moreover, applying the normal flow rule to Coulomb's law leads to overestimation of the divergence (Nedderman, 1992; Balendran and Nemat-Nasser, 1993; Tremblay and Mysak, 1997); and applying the coaxial flow rule with a constant dilatancy angle (Tremblay and Mysak, 1997) leads to an overall divergence which does not well capture the divergence when the mean compressive stress is high. The purpose of the present study is to propose a new constitutive law for pack ice, which is not only capable of simulating the in-plane shear and out-of-plane uniaxial compression processes, but also capable of avoiding overestimation of the divergence during shear.

\section{YIELD CURVE}

The motion of pack ice is traditionally described on a horizontal plane, where the motion and body forces are all vertically integrated (e.g. Gray and Morland, 1994):

$$
\rho h\left(\frac{\mathrm{d} \mathbf{u}}{\mathrm{d} t}+f \mathbf{k} \times \mathbf{u}\right)=\nabla \cdot \sigma+A \tau_{\mathrm{a}}+A \tau_{\mathrm{w}}-\rho h g \nabla H,
$$

where $\rho$ is the ice density, $A$ and $h$ the ice compactness and mean thickness, $\mathbf{k}$ a unit vector normal to the ice surface, $\mathbf{u}$ the ice velocity, $f$ the Coriolis parameter, $g$ the gravitational acceleration, $\tau_{\mathrm{a}}$ and $\tau_{\mathrm{w}}$ the wind and current shear stresses acting on and under the ice, $H$ the dynamic height of the sea surface, and $\sigma\left(\sigma_{i j}\right)$ is the two-dimensional internal ice stress.

For the stress tensor $\sigma_{i j}$, its invariants can be expressed by

$$
\sigma_{\mathrm{I}}=\frac{\sigma_{k k}}{2}, \quad \sigma_{\mathrm{II}}=\left(-\operatorname{det} \sigma_{i j}^{\prime}\right)^{1 / 2},
$$

where $\sigma_{i j}^{\prime}=\sigma_{i j}-\sigma_{1} \delta_{i j}$ is the stress deviator and $\delta_{i j}$ is the Kronecker operator. The principal stresses are

$$
\sigma_{1}=\sigma_{\mathrm{l}}+\sigma_{\| \mathrm{ll}} \quad \sigma_{2}=\sigma_{\mathrm{l}}-\sigma_{\mathrm{Il}} .
$$

Mathematically, the principal stresses are eigenvalues of $\sigma_{i j}$, which can be obtained by turning the $x-y$ axis counterclockwise with an angle of $\psi$, in which

$$
\tan 2 \psi=\frac{2 \sigma_{12}}{\sigma_{11}-\sigma_{22}} .
$$

As shown in Figure 1, the yield curve used in this paper consists of two parts: Coulomb's friction law describing the in-plane shear, and a maximum principal stress law describing the out-of-plane uniaxial compression. The Coulomb's friction law reads

$$
\sigma_{\mathrm{ns}}=-\sigma_{\mathrm{nn}} \tan \phi+c,
$$

where $\sigma_{\mathrm{ns}}$ and $\sigma_{\mathrm{nn}}$ are shear and normal stresses acting on the slip plane, $\phi$ is the angle of friction and $c$ is the cohesion. The minus sign before $\sigma_{\mathrm{nn}}$ is so taken as we define compressive stress as negative. According to the Mohr-Coulomb diagram 


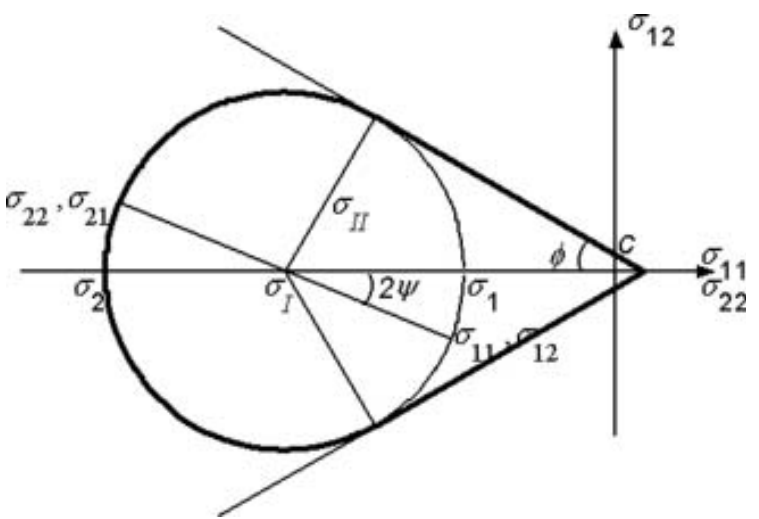

Fig. 1. Stress state in the Mohr-Coulomb diagram. The thick lines and curves show the yield curve used in the present constitutive law.

(Fig. 1), Equation (6) can be rewritten in terms of the stress invariants by

$$
F_{1}=\sigma_{\mathrm{II}}+\sigma_{\mathrm{I}} \sin \phi-c \cos \phi=0 .
$$

The maximum principal stress law describes a maximum compressive principal stress, that is $\sigma_{2} \geq-p^{*}$, where $p^{*}$ is the compressive strength under biaxial compression. According to Equation (4), this law can be expressed in terms of the stress invariants by

$$
F_{2}=\sigma_{\|}-\sigma_{1}-p^{*}=0 .
$$

The parameters involved in the yield curve are $p^{*}, \phi$ and $c$. The two-dimensional ice strength $p^{*}$ can be parameterized as a function of ice thickness and compactness (e.g. Hibler, 1979):

$$
p^{*}=P^{*} h \exp [-C(1-A)],
$$

where $P^{*}$ is the three-dimensional ice strength and $C$ is the reduction due to compactness.

The angle of friction can be determined through the relationship (e.g. Nedderman, 1992)

$$
\phi=\frac{\pi}{2}-\varphi
$$

where $\varphi$ is the smaller angle between the intersecting LKFs. Within compacted pack ice, a typical angle of $\varphi$ is about $30^{\circ}$ (e.g. Marko and Thomson, 1977; Kwok, 2000), resulting in a typical angle of friction of $60^{\circ}$.

Measurement of the cohesion $c$ in geophysical scales is very rare. In most cases, it is assumed to be 0 (e.g. Coon, 1974; Smith, 1983; Ip and others, 1991; Tremblay and Mysak, 1997). However, it may be important in numerical modeling of LKFs (e.g. Coon and others, 1998; Hibler and Schulson, 2000; Hopkins, 2000; Hutchings and Hibler, 2002). Therefore keeping this parameter in the present constitutive law is appropriate. In practice, it may be related to $p^{*}$ by

$$
c=\frac{p^{*}}{n}
$$

where $n$ is a constant, possibly ranging between 5 and 20 according to the field observation during the Sea Ice Mechanics Initiative (Coon and others, 1998).

The thick solid lines in Figure 2 show the yield curve as expressed in the coordinates of the stress invariants. For comparison, the cohesion $c$ has been set to 0 . Other similar yield curves shown in Figure 2 are: dash-dot lines together with the thick lines connecting to $-p^{*}$ (ice-cream cone) (Coon, 1974); thin solid curve (teardrop) (Rothrock, 1975);

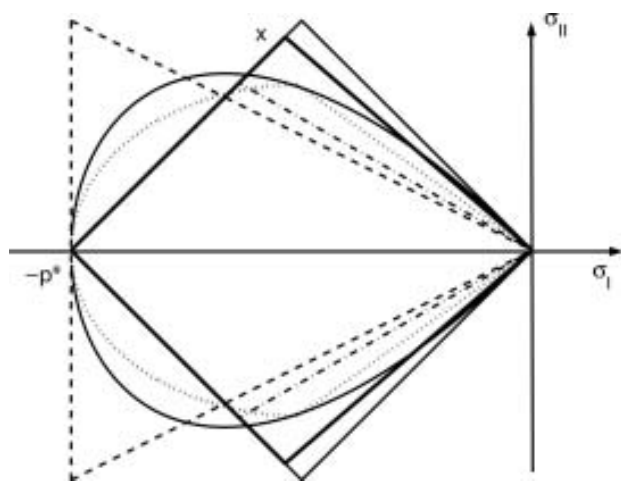

Fig. 2. Typical yield curves used in sea-ice dynamics in the coordinates of the stress invariants: dash-dot lines together with the thick lines connecting to $-p^{*}$ (ice-cream cone) (Coon, 1974); thin solid curve (teardrop) (Rothrock, 1975); thin solid lines (square) (Pritchard, 1981); dashed lines (Coulomb's law) (Tremblay and Mysak, 1997); dotted lines and curve (modified Coulomb's law) (Hibler and Schulson, 2000); and thick solid lines (diamond) (present study). For comparison the cohesion $c$ takes 0 and the slope angles are all taken from the original papers accordingly (see text for details).

thin solid lines (square) (Pritchard, 1981); dashed lines (Coulomb's law) (Tremblay and Mysak, 1997); and dotted lines and curve (modified Coulomb's law) (Hibler and Schulson, 2000). All the yield curves except the teardrop applied Coulomb's law. The angles of friction are all taken from the original papers, being $35^{\circ}$ (Coon, 1974), 90 (Pritchard, 1981), $30^{\circ}$ (Tremblay and Mysak, 1997), $45^{\circ}$ (Hibler and Schulson, 2000) and $60^{\circ}$ in the present study.

At the intersecting point of the present yield curve, $X$ (Fig. 2), we have

$$
\begin{aligned}
\sigma_{\mathrm{IX}} & =\frac{-p^{*}+c \cos \phi}{1+\sin \phi} \\
\sigma_{\mathrm{IIX}} & =\frac{p^{*} \sin \phi+c \cos \phi}{1+\sin \phi} .
\end{aligned}
$$

This point is the demarcation between the uniaxial compression and the Coulombic shear.

In the case of constant ice-strength parameters $\left(p^{*}, \phi\right.$ and $c$ ), expressing the yield curve in terms of the stress components $\sigma_{11}, \sigma_{22}$ and $\sigma_{12}$, we can obtain these components by solving together the momentum equation and the yield curve equations. However, when these parameters vary with ice conditions, we must consider the evolution of the velocity and mass fields simultaneously, as done in most dynamics models (e.g. Coon and others, 1974; Hibler, 1979).

\section{FLOW RULE}

The flow rule is the equation relating the stress tensor to the rate-of-strain tensor. Similar to what was done for the stress, for the rate-of-strain tensor $\dot{\varepsilon}_{i j}$ the invariants are

$$
\dot{\varepsilon}_{1}=\dot{\varepsilon}_{k k,} \quad \dot{\varepsilon}_{\| l}=2\left(-\operatorname{det} \dot{\varepsilon}_{i j}^{\prime}\right)^{1 / 2},
$$

where

$$
\dot{\varepsilon}_{i j}^{\prime}=\dot{\varepsilon}_{i j}-\dot{\varepsilon}_{1} \delta_{i j} / 2
$$

is the rate-of-strain deviator. The principal rates of strain are

$$
\dot{\varepsilon}_{1}=\frac{\dot{\varepsilon}_{1}+\dot{\varepsilon}_{\| I}}{2}, \quad \dot{\varepsilon}_{2}=\frac{\dot{\varepsilon}_{1}-\dot{\varepsilon}_{\| I}}{2} \text {. }
$$



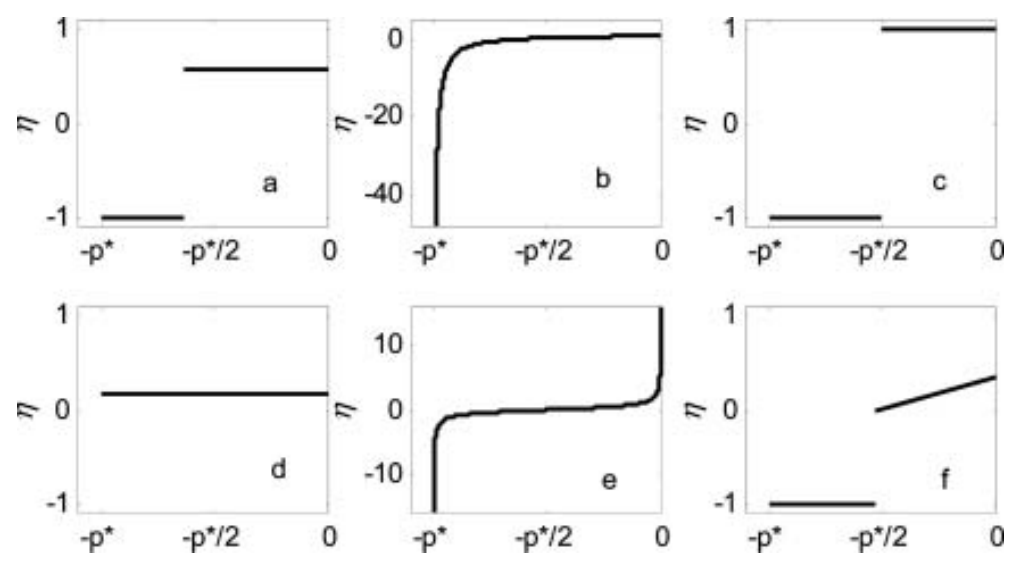

Fig. 3. The ratios of divergence to shear, $\eta=\dot{\varepsilon}_{1} / \dot{\varepsilon}_{\mathrm{ll}}$, for different yield curves and flow rules: (a) yield curve of ice cream cone with the normal flow rule (Coon, 1974); (b) teardrop yield curve with the normal flow rule (Rothrock, 1975); (c) square yield curve with the normal flow rule (Pritchard, 1981); (d) Coulomb's law with the coaxial flow rule (Tremblay and Mysak, 1997); (e) modified Coulomb's law with a combined normal and non-normal flow rule (Hibler and Schulson, 2000); and (f) diamond yield curve with a combined normal and coaxial flow rule (present study).

These principal rates of strain can similarly be obtained by turning the $x-y$ coordinates counterclockwise with an angle of $\Phi$, where

$$
\tan 2 \Phi=\frac{2 \dot{\varepsilon}_{12}}{\dot{\varepsilon}_{11}-\dot{\varepsilon}_{22}}
$$

For granular materials the Lévy-Mises flow rule, also known as the coaxial flow rule, is often applied (e.g. Nedderman, 1992). This flow rule states that the strain-rate and stress deviators are proportional:

$$
\dot{\varepsilon}_{i j}^{\prime}=\lambda^{\prime} \sigma_{i j}^{\prime}
$$

where $\lambda^{\prime}$ is a scalar variable. This condition leads to $\Phi=\psi$, that is, the principal stresses and the principal rates of strain are coaxial. The disadvantage of this flow rule, however, is that it does not provide a means to estimate the divergence. On the other hand, the normal flow rule is often applied to the sea-ice dynamics (e.g. Coon and others, 1974; Hibler, 1979; Pritchard, 1981). The normality can be expressed by

$$
\dot{\varepsilon}_{i j}=\lambda \frac{\partial F}{\partial \sigma_{i j}}=\frac{\lambda}{2}\left(\frac{\partial F}{\partial \sigma_{l}} \delta_{i j}+\frac{\sigma_{i j}^{\prime}}{\sigma_{\| l}} \frac{\partial F}{\partial \sigma_{\| l}}\right),
$$

where $\lambda$ is a non-negative scalar variable and $F$ is the yield curve. Comparing Equations (16) and (17) we can see that the normal flow rule automatically possesses the coaxial property; it is therefore a special case of the coaxial flow rule.

For the ice stress on the yield curve of uniaxial compression, as shown in Equation (8), applying the normal flow rule leads to

$$
\dot{\varepsilon}_{1}=-\dot{\varepsilon}_{\| 1}
$$

This result shows that the ice is undergoing uniaxial compressive deformation, producing ice ridges perpendicular to the compressive stress. Such a deformation pattern is physically reasonable. Therefore, we can take the normal flow rule to describe the uniaxial compressive flow.

Similarly, applying the normal flow rule to Coulomb's friction law, as shown in Equation (7), we have

$$
\dot{\varepsilon}_{1}=\dot{\varepsilon}_{||} \sin \phi .
$$

In general, such divergence is overestimated (e.g. Nedderman, 1992; Tremblay and Mysak, 1997). As a result, the normal flow rule does not apply in this case. Following Balendran and Nemat-Nasser (1993), an angle of dilatancy $\delta$ can be employed to replace $\phi$, which gives

$$
\dot{\varepsilon}_{1}=\dot{\varepsilon}_{\mid l} \sin \delta \text {. }
$$

The angle of dilatancy $\delta$ here is generally less than $\phi$. As a consequence, the normality is no longer fulfilled. In addition, as a dense sample the compacted pack ice would normally possess a positive $\delta$ (Balendran and Nemat-Nasser, 1993). Then a continuous dilation leads to a continuous decrease of the ice compactness and $\delta$. When the stress ratio $\sigma_{\mathrm{II}} / \sigma_{\mathrm{I}}$ reaches the peak (point $\mathrm{X}$ in Fig. 2), the critical stress state appears, where the dilatancy becomes 0 . In such a case, $\delta$ may be parameterized along the yield curve of Coulomb's shear such that

$$
\delta=\frac{\sigma_{\mathrm{I}}-\sigma_{\mathrm{IX}}}{c \cot \phi-\sigma_{\mathrm{IX}}} \delta_{\mathrm{m}}
$$

where $\delta_{\mathrm{m}}$ is the maximum angle of dilatancy. It may take $20^{\circ}$, with $10^{\circ}$ (Tremblay and Mysak, 1997) as a mean.

Figure 3 shows the ratios of divergence to shear, $\eta=\dot{\varepsilon}_{1} / \dot{\varepsilon}_{\| 1}$, for different yield curves and flow rules. As can be seen, the ratios from Coon's cream cone and Pritchard's square are rather close to that of the present study, consisting of a uniaxial compression and shear. The difference in these three constitutive laws lies in how much divergence would occur during shear. Tremblay and Mysak's constitutive law, as has been pointed out, takes an overall divergence when the compressive stress is higher than $-p^{*}$. Rothrock's teardrop with the normal flow rule possesses a small divergence when $\sigma_{\mathrm{I}}$ is low, but results in significant convergence when $\sigma_{\mathrm{I}}$ becomes close to $-p^{*}$. Hibler and Schulson's constitutive law generally yields a small ratio of divergence to shear, but it tends to be quite large when $\sigma_{1}$ approaches 0 or $-p^{*}$. As suggested by Equation (1), the teardrop and modified Coulomb's law hardly result in uniaxial pressure ridges, which implies that in the case of high mean compressive stress these two constitutive laws are less effective than the others. And using a constant divergence rate in Tremblay and Mysak's constitutive law does not predict compression except when the mean compressive stress reaches $-p^{*}$, which is physically unreasonable. For the constitutive laws applied by Coon (1974) and Pritchard (1981), we know that the typical angle of friction needs to be about $60^{\circ}$ and that the normal flow rule usually overestimates divergence during 
shear. Therefore, the present constitutive law possesses the highest capability in modeling the observed LKF and divergence patterns. Further determination of the ratio of divergence to shear can be achieved by checking the available satellite observations (e.g. Kwok, 2000).

The flow rule on the three corners in the present constitutive law needs some special treatment. Basically, the corner where $\sigma_{1}=0$ can be seen as a pure divergence case, while the corner where $\sigma_{1}=-p^{*}$ can be seen as a pure convergence case. At the intersecting point $X$ (Fig. 2), the maximum shear stress reaches the peak, and the flow rule can be regarded as a pure shear case. Such treatment is consistent with the classical Tresca yield curve with the normal flow rule.

With the coaxial flow rule, the components of the rate of strain can be expressed by

$$
\begin{aligned}
& \dot{\varepsilon}_{11}=\frac{\partial u}{\partial x}=\frac{1}{2}\left(\dot{\varepsilon}_{\mid}+\dot{\varepsilon}_{||} \cos 2 \psi\right) \\
& \dot{\varepsilon}_{22}=\frac{\partial v}{\partial y}=\frac{1}{2}\left(\dot{\varepsilon}_{\mid}-\dot{\varepsilon}_{||} \cos 2 \psi\right) \\
& \dot{\varepsilon}_{12}=\frac{1}{2}\left(\frac{\partial u}{\partial y}+\frac{\partial v}{\partial x}\right)=\frac{1}{2} \dot{\varepsilon}_{||} \sin 2 \psi,
\end{aligned}
$$

where $u$ and $v$ are the two-dimensional ice velocity components. Combining Equations (15), (18)/(20) and (22) gives two equations describing the evolution of $u$ and $v$, from which the velocity field can be obtained, provided the boundary conditions are given.

\section{CONCLUDING REMARKS}

A new constitutive law is presented to describe the plastic behavior of pack ice, as shown in Equations (7), (8), (16), (17), (20) and (21). The yield curve consists of Coulomb's friction law describing the in-plane shear, and the maximum principal stress law describing the out-of-plane uniaxial compression. For the shear deformation, the coaxial flow rule with a parameterized dilatancy is proposed, while for the uniaxial compression the normal flow rule is shown to be appropriate. This constitutive law is not only capable of simulating the in-plane shear and out-of-plane uniaxial compression, but also capable of avoiding overestimation of divergence during shear.

A comparison of the present constitutive law with other similar laws shows that the present constitutive law possesses the highest capability in modeling the observed LKF and divergence patterns. This law has physical significance in forming the pressure ridges and intersecting LKFs, resulting in a typical angle of $30^{\circ}$ between the intersecting LKFs.

\section{ACKNOWLEDGEMENTS}

I am extremely grateful to $M$. Leppäranta and two anonymous reviewers, whose comments greatly improved the manuscript. This work was partly supported by the Chinese National Natural Science Foundation under contract 40233032 and by the Maj and Tor Nessling Foundation of Finland. The Chancellor's Travel Grant of the University of Helsinki is also gratefully acknowledged.

\section{REFERENCES}

Balendran, B. and S. Nemat-Nasser. 1993. Double sliding model for cyclic deformation of granular materials, including dilatancy effects. J. Mech. Phys. Solids, 41(3), 573-612.

Coon, M.D. 1974. Mechanical behavior of compacted Arctic ice floes. J. Pet. Technol., 26, 466-470.

Coon, M.D., G.A. Maykut, R.S. Pritchard, D.A. Rothrock and A.S. Thorndike. 1974. Modeling the pack ice as an elasticplastic material. AIDJEX Bull. 24.

Coon, M.D., G.S. Knoke, D.C. Echert and R.S. Pritchard. 1998. The architecture of an anisotropic elastic-plastic sea ice mechanics constitutive law. J. Geophys. Res., 103(C10), 21,915-21,925.

Erlingsson, B. 1988. Two-dimensional deformation patterns in sea ice. J. Glaciol., 34(118), 301-308.

Gray, J.M.N.T. and L.W. Morland. 1994. A two-dimensional model for the dynamics of sea ice. Philos. Trans. R. Soc. London, Ser. B, 347(1682), 219-290.

Hibler, W.D., III. 1979. A dynamic thermodynamic sea ice model. J. Phys. Oceanogr., 9(7), 815-846.

Hibler, W.D., III and E.M. Schulson. 2000. On modeling the anisotropic failure and flow of flawed sea ice. J. Geophys. Res., 105(C7), 17,105-17,120.

Hopkins, M.A. 2000. The effect of tensile strength in the Arctic ice pack. In Dempsey, J.P., H.T. Shen and L.H. Shapiro, eds. IUTAM Symposium on Scaling Laws in Ice Mechanics and Ice Dynamics, 13-16 June 2000, University of Alaska Fairbanks. Proceedings. Dordrecht, Kluwer Academic Publishers, 373-386.

Hutchings, J.K. and W.D. Hibler, III. 2002. Modelling sea ice deformation with a viscous-plastic isotropic rheology. In Squire, V.A. and P.J. Langhorne, eds. Proceedings of the 16th IAHR International Symposium on Ice, 2-6 December 2002, Dunedin, New Zealand, Vol. 2. Dunedin, International Association of Hydrological Engineering and Research, 358-366.

Ip, C.F., W.D. Hibler, III and G.M. Flato. 1991. On the effect of rheology on seasonal sea-ice simulations. Ann. Glaciol., 15, $17-25$.

Kwok, R. 2000. Deformation of the Arctic Ocean sea ice cover between November 1996 and April 1997: a survey. In Dempsey, J.P., H.T. Shen and L.H. Shapiro, eds. Scaling laws in ice mechanics and ice dynamics. Boston, MA, Kluwer Academic Publishers, 315-323.

Marko, J.R. and R.E. Thomson. 1977. Rectilinear leads and internal motions in the ice pack of the western Arctic Ocean. J. Geophys. Res., 82(6), 979-987.

Nedderman, R.M. 1992. Statics and kinematics of granular materials. Cambridge, Cambridge University Press.

Pritchard, R.S. 1981. Mechanical behaviour of pack ice. In Selvadurai, A.P.S., ed. Mechanics of structured media. New York, Elsevier, 371-405.

Pritchard, R.S. 1988. Mathematical characteristics of sea ice dynamics models. J. Geophys. Res., 93(C12), 15,609-15,618.

Rothrock, D.A. 1975. The energetics of the plastic deformation of pack ice by ridging. J. Geophys. Res., 80(33), 4514-4519.

Smith, R.B. 1983. A note on the constitutive law for sea ice. J. Glaciol., 29(101), 191-195.

Tremblay, L.B. and L.A. Mysak. 1997. Modelling sea ice as a granular material, including the dilatancy effect. J. Phys. Oceanogr., 27(11), 2342-2360.

Wang, K. 2004. Preliminary results of yield criteria of pack ice and their impact on the orientation of linear kinematic features. In Proceedings of the 17th IAHR International Symposium on Ice, 21-25 June 2004, St. Petersburg, Russia, Vol. 1. St Petersburg, International Association of Hydrological Engineering and Research, 135-143. 